\title{
Interleukin-17 - association to silent lupus nephritis and disease activity
}

\author{
Adriana Metoni ${ }^{1,2}$, Paul Balanescu ${ }^{1,3}$, Alina Dima ${ }^{1,3}$, Eugenia Balanescu ${ }^{1,3}$, Camelia Badea $^{1,3}$, \\ Razvan Ionescu ${ }^{1,3}$, Carmen C. Diaconu ${ }^{4}$, Cristian Baicus ${ }^{1,3}$, Dumitru Matei ${ }^{1,5}$
}

${ }^{1}$ Carol Davila University of Medicine and Pharmacy, Bucharest, Romania,

Individual medical practice, Family Medicine, CMI Metoni Adriana

${ }^{3}$ Colentina Research Center, Colentina Clinical Hospital, Bucharest, Romania

${ }^{4}$ Stefan S. Nicolau Institute of Virology, Bucharest, Romania

${ }^{5}$ Prof. Alfred Russescu Institute of Mother and Child Bucharest, Romania

\author{
Corresponding author: \\ Adriana Metoni, MD \\ Carol Davila University of Medicine \\ and Pharmacy, Bucharest, Romania \\ E-mail: motca_adriana@yahoo.com
}

\section{ABSTRACT}

Background: Systemic lupus erythematosus is a multiorganic, chronic immune disease and lupus nephritis, a severe manifestation, represents the strongest predictor of a poor outcome of this pathology. Cytokines play an important role in lupus nephritis and consequently, their use as biomarkers of active systemic lupus erythematosus disease is of particular interest. The purpose of this work was to study the pro-inflammatory role of interleukin-17 in renal involvement in patients with systemic lupus erythematosus (SLE).

Methods: We performed a retrospective study of 87 patients diagnosed with SLE according to the Systemic Lupus International Collaborating Clinics 2012 diagnosis criteria. In this study, we determined the serum levels of interleukin-17 by ELISA.

Results: It was observed that 49 patients in the study group presented with positive values of interleukin-17, range $(1.12-23.66) \mathrm{pg} / \mathrm{ml}$. There was a positive correlation of interleukin-17 with active SLE as assessed by the Systemic Lupus Erythematous Disease Activity Index. No association was found between serum interleukin-17 level and renal pathology at the inclusion or in the clinical history of the patients. Patients with leukocyturia and hematuria presented higher values of serum interleukin-17 than those without these manifestations. In the linear regression model, after adjusting for age, gender and treatment we found an independent association between serum IL-17 levels and leukocyturia presence with $\mathrm{OR}=2.06,95 \% \mathrm{Cl}$ range (1.22-2.89)

Conclusions: A positive correlation has been observed between serum IL-17 and the SLE disease activity as assessed by the SLEDAI score computed without anti-DNA antibodies. Also, the IL-17 levels was strongly associated with the presence of leukocyturia and hematuria, even in patients with no clinical evidence of renal disease that might have silent lupus nephritis usually associated with a benign renal outcome.

Key words: systemic lupus erythematosus, lupus nephritis, interleukin-17, leukocyturia, hematuria

\section{INTRODUCTION}


disease characterized by heterogeneous, multisystem involvement and the production of a large array of autoantibodies (1).

Renal involvement is common in SLE. Many patients presenting at the time of diagnosis of SLE, lupus nephritis (LN), an abnormal urinalysis associated sometimes with an increased concentration of serum creatinine. A large amount of clinical, immunopathological and experimental data support the hypothesis that glomerolonephritis have an immunological determinism $(2,3)$.

The pattern of glomerular injury seen in SLE is primarily related to the site of formation of the immune deposits, which are located in the mesangium, subendothelial and/or sub-epithelial compartments of the glomerulus.

The immune deposits consist mainly of anti-doublestranded DNA antibodies (anti-ds DNA or anti-DNA) directed against nucleosomes. Some antibodies may bind directly to components of glomerular basement membrane and mesangium $(4,5)$.

Two immune mechanisms are responsible for glomerular injury: an inflammatory and a noninflammatory mechanism. When inflammatory mechanism is involved, like in glomerulonephritis, the immune deposits are localized in mesangium and sub-endothelial space, proximal to the glomerular basement membrane (GMB), and are therefore in communication with the vascular space. These deposits can activate the complement with generation of chemo attractants $\mathrm{C} 3 \mathrm{a}, \mathrm{C} 5 \mathrm{a}$, resulting in the influx of neutrophils and mononuclear cells. These changes appear histologically as a mesangial, focal or diffuse proliferative glomerulonephritis and clinically by an active urine sediment (red cells, white cells, cellular and granular casts), proteinuria and often an acute decline of renal function.

The deposits in sub-epithelial space can also activate complement; there is no influx of inflammatory cells since the chemoattractants are separated from the circulation by the GMB. This immune non-inflammatory glomerular lesion has as clinical expression proteinuria and the nephrotic syndrome with little or no hematuria and no red blood cell casts.

Glomerular injury of immune origin is mediated by the actions of components of both the innate and adaptive immune system.

Cytokines play an important role in LN, so use of cytokines as biomarkers of disease activity in SLE and $L N$ is of particular interest (6).

Recent studies indicate a central role of interleukin-17 (IL-17) in the pathogenesis of lupus nephritis (7). IL-17 is a prototypical T helper-17 (Th-17) cells subset evolved from CD4+ T cells under the influence of interleukin-6 (IL-6), interleukin-21 (IL-21) and interleukin-1 (IL-1). It is known that this cytokine has a strong pro-inflammatory effect, induces other cytokines synthesis, promotes recruitment of inflammatory cells and facilitates T-cells infiltration (8).

The purpose of this work was to study the correlation of IL-17 with the renal involvement in SLE patients.

\section{MATERIAL AND METHODS}

\section{Clinical assessment of the patients}

The study included 87 patients, with the age over 18 years, diagnosed with SLE based on the Systemic Lupus International Collaborating Clinics (SLICC) 2012 diagnosis criteria (9). Patients were hospitalized between January and December 2014 in Internal Medicine Clinic of Colentina Clinical Hospital Bucharest, Romania. Written informed consent was obtained from all subjects. The clinical and biological data were gathered retrospectively.

We have established baseline variables: age, sex, age body mass index, smoking, hypertension, standard therapy and treatment duration at the moment of inclusion in the study.

Disease activity was assessed on the basis of Systemic Lupus Erythematosus Disease Activity Index (SLEDAI). Also, a SLEDAl score without the data for the anti-DNA antibodies was computed (10).

\section{Biological assessment of the patients}

Data regarding the erythrocyte sedimentation rate (ESR), C-reactive protein (CRP), hemoglobin, erythrocytes, leukocytes, lymphocytes, creatinine, anti-dsDNA antibodies and the urinalysis (leucocyturia, hematuria, proteinuria) were collected from the patients' clinical files.

\section{Method of determination of serum IL-17 (Serum cytokine quantification)}

Serum IL-17 was determined using a commercial ELISA kit (Human IL-17 Quantikine ELISA Kit, R\&D Systems, Minneapolis, MN, USA), according to manufacturer instructions (http://www.rndsystems.com/ Products/D1700) on samples stored at $-70^{\circ} \mathrm{C}$ in the Immunology Laboratory of Colentina Research Center, Bucharest. All samples were analyzed in duplicate and mean optical density at $450 \mathrm{~nm}$ was considered for evaluation of IL-17 concentrations after interpolation on a standard curve. 


\section{Statistical analysis}

Continuous variables were presented as mean \pm standard deviation if the distribution was normal and as median, minimum and maximum values if the distribution was not normal. Fischer's exact test was used in order to evaluate whether two categorical variables were associated. Non-parametric Mann Whitney $U$ tests was used to assess the differences between serum concentrations of the cytokine. Spearman's rank correlation coefficient was used in order to determine if there were linear correlations between continuous variables. Independent association between variables, was assessed by linear regression models performed with serum IL-17 levels as dependent variables and relevant clinical and paraclinical features as independent variables. Statistical significance was set at the level of $p$ $<0.05$. Statistical analysis was performed using SPSS, version 16 for Windows.

\section{RESULTS}

The distribution of SLE patients according to sex and age was as follows: female 76 patients $(87.6 \%)$ and median (inf;max) age was $45(20 ; 78)$ years. 16 of the SLE patients (18.4\%) taken in the study, presented with renal involvement at the time of recruitment and 37 patients (42.5\%) had renal impairment in their clinical history.

Clinical and laboratory investigation results are summarized in table 1.

We observed a highly significant association between higher SLEDAl in renal impaired at recruitment or in clinical history of the patients with SLE $(p<0.001$ in both situations).

49 patients (56.3\%) had a positive value of serum concentration of interleukin-17 (IL-17) and the mean IL-17 concentration was $3.71 \mathrm{pg} / \mathrm{mL}$. Median (inf;max) serum IL-17 was $2.25(0 ; 23.66 \mathrm{pg} / \mathrm{ml})$. Serum IL-17 was undetectable in 38 patients.

We observed a positive correlation between serum IL-17 and SLE disease activity assessed by SLEDAI without anti-DNA ( $r=0.178 ; p=0.03)$ and marginal correlation with the SLE disease activity as assessed by SLEDAl score ( $r=0.178 ; p=0.063$ ).

No association was found between serum IL-17 levels and renal involvement at the time of recruitment or in the clinical history of the patients. There was no correlation between serum IL-17 and anti-dsDNA antibodies, CRP and ESR.

Patients with leukocyturia and hematuria had higher concentration of serum IL-17 compared to patients with absence of leukocyturia and hematuria -
Table 1. Baseline characteristics of the SLE patients

\begin{tabular}{|c|c|}
\hline Characteristic & \\
\hline Female gender, no (\%) & $76(87.6 \%)$ \\
\hline Age, years & $45(20-78)$ \\
\hline Renal impairment, no(\%) & $16(18.4 \%)$ \\
\hline Cutaneous impairment & $26(29.9 \%)$ \\
\hline Articular impairment & $29(33.3 \%)$ \\
\hline Serositis & $3(3.4 \%)$ \\
\hline Neurological impairment & $6(69 \%)$ \\
\hline Hematological impairment & $33(37.9 \%)$ \\
\hline Leukocyturia & $23(27.4 \%)$ \\
\hline Hematuria & $11(12.6 \%)$ \\
\hline $\mathrm{ESR}(\mathrm{mm} / \mathrm{h})$ & $19(2-95)$ \\
\hline $\mathrm{CRP}(\mathrm{mg} / \mathrm{dL})$ & $2.36(0-35.42)$ \\
\hline DNA ds antibodies (UI/L) & $70.7(5.6-1615)$ \\
\hline Urea (mg/dL) & $34(18.7-115)$ \\
\hline Creatinine $(\mathrm{mg} / \mathrm{dL})$ & $0.73(0.42-3.89)$ \\
\hline Proteinuria/24h (mg/L), med(min;max) & $79.5(0.0 ; 4900.0)$ \\
\hline SLEDAl, med(min;max) & $6(0.0 ; 48.0)$ \\
\hline SLEDAI without complement & $6(0-46)$ \\
\hline Hemoglobin $(\mathrm{g} / \mathrm{dL})$, med(min;max) & $12.6(6.8 ; 16.6)$ \\
\hline
\end{tabular}

$6.76(0-23.66) \mathrm{pg} / \mathrm{mL}$ versus $0 \mathrm{pg} / \mathrm{mL}(0-15.77$ $\mathrm{pg} / \mathrm{mL}), p<0.001$, patients with only leukocyturia 3.38 $\mathrm{pg} / \mathrm{mL}(0-23.66 \mathrm{pg} / \mathrm{mL})$ versus $1.12 \mathrm{pg} / \mathrm{mL}(0-19.15$ $\mathrm{pg} / \mathrm{mL}) \mathrm{p}=0.05$ patients with only hematuria).

Leukocyturia and hematuria were present in 23 patients (47\%) from the IL-17 positive group (49 patients) with positive value of serum IL-17; 10 (43.5\%) patients had just leukocyturia, 7 patients (30.4\%) had leukocyturia and hematuria, 4 (17.4\%) patients had leukocyturia and proteinuria, 2 patients (8.7\%) had leukocyturia, hematuria, proteinuria, and 3 patients had urinary infection.

In the linear regression model, after adjusting for age, gender and treatment there was an independent association between serum IL-17 levels and leukocyturia presence (OR=2.06, 95\% Cl 1.22-2.89).

\section{DISCUSSION}

Several studies have shown a significant increase of IL-17 in patients with SLE, a multi-organ chronic immune disease. IL-17 is a cytokine with a powerful pro-inflammatory activity and IL-17 producing $T$ cells are present in the kidneys of patients with lupus nephritis (LN) $(11,12)$.

Different authors reported in their studies significantly higher levels of serum IL-17 in patients with SLE than in controls (11).

In our study conducted on 87 patients with SLE it was observed that 49 patients presented positive 
values of IL-17 concentrations while 38 patients had no detectable serum level of IL-17. Likewise, we found a correlation between IL-17 and SLE disease activity assessed by SLEDAI without anti-DNA antibodies and a trend for correlation with SLE disease activity assessed by SLEDAI. Conversely, other studies showed no correlations in any patient subset $(11,12,13)$.

In this study we could not find any correlation between IL-17 and renal impairment at the time of recruitment or in the clinical history of these patients, and also with anti-dsDNA antibodies, ESR and CRP. We mention that at baseline, 16 patients had renal involvement and 37 had renal impairment in their history and they received corticosteroids or immunosuppressant therapy. There was no association between IL-17 and type of treatment or treatment duration. Previously, it was reported that IL-17-producing cells are present in the inflamed kidney tissues of the patients with lupus nephritis (7).

The reasons for these discrepancies are unknown; uncontrolled factors such as corticosteroids or immunosuppressant therapy may influence serum cytokine levels. Likewise, serum IL-17 levels may not be an accurate reflection of total IL-17 production. IL-17 may be localized in inflamed tissue in some cases, such as renal tissue, so the serum IL-17 levels may not be a faithful reflection of its endogenous production.

Various studies found that serum IL-17 expression level was associated with the severity of lupus nephritis, which evaluated IL-17 presence by histopathologic analysis of the kidney sections and urine protein levels $(14,15)$.

Xing et al., however, argued that the serum expression of IL-17 levels in LN patients exhibited a significant increase compared to patients without nephritis and healthy controls (16).

In our study we found 23 patients with an interesting correlation between IL-17 and leukocyturia and hematuria without proteinuria. This correlation may be the result of mild or moderate renal impairment that associated just active urinary sediment (leukocyturia and hematuria).

The mechanism of renal impairment is determined by immune inflammation expressed in mesangial, focal or diffuse proliferative glomerulonephritis. The renal disease in patients with silent lupus nephritis often remains clinically silent and is associated with a benign renal outcome. Renal histopathology by renal biopsy could help support this hypothesis.

\section{CONCLUSION}

In this study, a positive correlation has been observed between serum IL-17 and the SLE disease activity as assessed by the SLEDAl score computed without anti-DNA antibodies. Also, the IL-17 levels was strongly associated with the presence of leukocyturia and hematuria, even in patients with no clinical evidence of renal disease. These patients might have silent lupus nephritis that is associated with a benign renal outcome.

\section{Acknowledgments}

This paper is supported by the Sectorial Operational Programme Human Resources Development (SOP HRD), financed from the European Social Fund and by the Romanian Government under the contract number POSDRU/159/1.5/S/137390

\section{Potential conflict of interest}

Nothing to report.

\section{Disclosure}

All authors contributed equally.

\section{REFERENCES}

1. Jinoos Yazdany and Maria Dall'Era. Definition and Classification of Lupus And Lupus - Related Disorder. In: Wallace D, Hannahs Hann B (eds), DUBOIS' Lupus Erythematosus and Related Syndromes, Eighth Edition. United States: Elservier- Health Sciences Division; 2012.

2. Mathieson PW. Glomerulonephritis. Semin. Immunopathol. 2007 Nov; 29(4): 315-16

3. Nangaku M, Couser WG. Mechanism of immune-deposit formation and the mediation of immune renal injury. Clin. Exp. Nephrol. 2005 Sep;9(3):183-91.

4. Krishnan MR, Wang C, Marion TN. Anti-DNA autoantibodies initiate experimental lupus nephritis by binding directly to the glomerular basement membrane in mice. Kidney Int. $2012 \mathrm{Jul} ; 82(2): 184-92$.

5. Lech M, Anders HJ. The pathogenesis of lupus nephritis. J Am Soc Nephrol. 2013 Sep;24(9):1357-66.

6. Yap DY, Lai KN. Cytokines and their roles in the pathogeneis of Systemic Lupus Erythematosus: from basics to recent advances. J Biomed Biotechnol. 2010 May;2010(365083):1-10.

7. Apostolidis SA, Crispin JC, Tsokos GC. IL-17-producing T cells in lupusnephritis. Lupus. 2011 Feb ;20(2):120-4.

8. Zhang Z, Kyttaris VC, Tsokos GC. The role of IL-23/LL-17 axis in lupus nephritis. J Immunol. 2009 Sep 1;183(5):3160-9.

9. Tan EM, Cohen AS, Fries JF, Masi AT, McShane DJ, Rothfield NF, Schaller JG, Talal N, Winchester RJ. The 1982 revised criteria for the classification of systemic lupus erythematosus. Arthritis Rheum. 1982 Nov; 25(11): 1271-7.

10. Gladman DD, Ibanez D, Urowitz MB. Systemic lupus erythematosus disease activity index 2000. J Rheumatol. 2002 Feb; 29:288-291.

11. Fabien BV, Northcott M, Hoi A, Mackay F and Morand EF. Clinical associations of interleukin-17 in systemic lupus erythematosus. Arthritis Research and Therapy 2013 Aug;15(4)R97, 1-9.

12. Zhao, Xue-Fei; Pan, Hai-Feng; Yuan, Hui; Zhang, Wen-Hui; Li, Xiang-Pei; Wang, Gui-Hong; Wu, Guo-Cui; Su, Hong; Pan, Fa-Ming; Li, Wen-Xian; Li, 
Lian-Hong; Chen, Guo-Ping; Ye, Dong-Qing . Increased serum interleukin17 in patients with systemic lupus erythematosus. Molecular Biology Reports 2010 Jan;37(1):81-5.

13. Mok Mo Yin, Wu Hai Jing, Lo Yi, Lau, Chak Sing. The relation of interleukin-17(IL-17) and IL-23 to Th1/Th2 cytokines and disease activity in systemic lupus erythematosus. Journal of Rheumatology. 2010 0ct;37(10):2046-52.

14. Zickert $A$, Amoudruz $P$, Sundström $Y$, Rönnelid J, Malmström $V$, Gunnarsson I. II-17 and IL-23 in lupus nephritis - association to histopathology and reponse to treatment. BMC Immunol. 2015 Feb 12;16:7. doi: 10.1186/s12865-015-0070-7.

15. Wen Zhenke, Xu Lin, Xu Wei, Yin Zhinan, Gao Xiaoming, Xiong Sidong. Interleukin-17 Expression Positively Correlate with Disease Severity of
Lupus Nephritis by Increasing Anti-Double Stranded DNA Antibodies Production in a Lupus Model Induced by Activated Lymphocytes Derived DNA . PLoS ONE. 2013 Mar;8(3):e58161, 1-10.

16. Miyake $\mathrm{K}$, Akahoshi M, Nakashima $H$. Th-subset balance in lupus nephritis. J Biomed Biotechnol. 2011;2011:980286. doi: 10.1155/2011/ 980286. Epub 2011 Aug 28.

17. Xing Q1, Wang B, Su H, Cui J, Li J. Elevated Th-17 cells are accompanied by FoxP3 + Treg cells decrease in patients with lupus nephritis. Rheumatol Int. 2012 Apr;32(4):949-58. doi: 10.1007/s00296-010-1771-0. Epub 2011 Jan 18.

18. Chen D-Y, Chen Y-M, Wen M-C, Hsieh T-Y, Hung W-T, Lan J-L. The potential role of Th 17 cells and the Th17- related cytokines in the pathogenesis of lupus nephritis. Lupus. 2012 Nov;21(13):1385-96. 